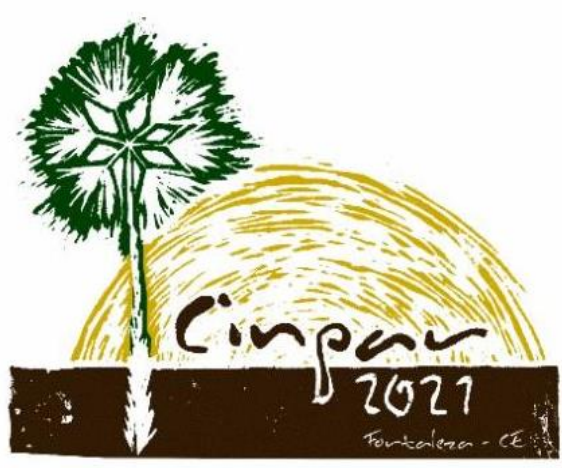

XVII Congresso Internacional sobre Patologia e

Reabilitação das Construções

XVII Congreso Internacional sobre Patología y Rehabilitación de las Construcciones

XVII International Conference on Pathology and Constructions Rehabilitation

FORTALEZA (Brasil), 3 a 5 de junho de 2021

https://doi.org/10.4322/CINPAR.2021.063

\title{
Utilização da termografia na identificação de anomalias exógenas e funcionais no patrimônio histórico de Curvelo/MG
}

\section{Use of thermography in the identification of exogenous and functional anomalies in the historical heritage of Curvelo/MG} \author{
Pena BORTONE ${ }^{4}$, Rachel Jardim MARTINI \\ ${ }^{1}$ CEFET/MG, Curvelo, Brasil, pedrohebrique137@gmail.com \\ ${ }^{2}$ CEFET/MG, Curvelo, Brasil, anaclaraons36@gmail.com \\ ${ }^{3}$ CEFET/MG, Curvelo, Brasil, marcos.ramos@cefetmg.br \\ ${ }^{4}$ CEFET/MG, Curvelo, Brasil, thiago.bortone@ cefetmg.br \\ ${ }^{5}$ CEFET/MG, Curvelo, Brasil, martini@cefetmg.br
}

Pedro Henrique Leite SOARES ${ }^{1}$, Ana Clara Oliveira Nascimento SILVEIRA ${ }^{2}$, Marcos de Paulo RAMOS 3 , Thiago

\begin{abstract}
Resumo: A salvaguarda do patrimônio histórico-cultural é um interesse comum da sociedade, visto que ele expressa a identidade cultural e econômica local. Na região do Município de Curvelo/MG, os edifícios são derivados da base de transporte férreo do século passado, que trouxe significativa evolução sócio econômica para a região. Manter essas estruturas históricas em um bom estado de conservação representa desafios inerentes às políticas de conservação, além de conhecimentos específicos de materiais e técnicas construtivas retrospectivas. $\mathrm{O}$ objetivo deste trabalho é identificar manifestações patológicas utilizando a termografia na caracterização do patrimônio histórico da região de Curvelo/MG, particularmente o Prédio 2, conhecido como Lúcio Cardoso, que pertence ao conjunto urbano da Praça Central do Brasil. A análise de edificações de importância histórica deve ser precedida de um planejamento detalhado. A proposta metodológica consiste na aplicação de um ensaio não-destrutivo (END), nomeadamente a técnica da termografia. Esta tem como objetivo auxiliar na vistoria predial, ressaltar as anomalias existentes na edificação analisada, assim como identificar suas características construtivas e possíveis reformas ou alterações arquitetônicas. Com a termografia é possível observar algumas anomalias como fissuras, infiltrações, vazamentos, deslocamento de revestimentos ou pintura, desagregação de paredes, entre outras. Para este estudo de caso também foi feito o levantamento de informações e coletas de dados visuais, levantamentos de laudos anteriores, revisões bibliográficas e análise histórica arquitetônica da edificação. Como resultado, verificou-se com a vistoria e a análise visual que a edificação está em processo de reforma e encontra-se em bom estado de conservação, apesar de não ter sido preservada na integralidade, suas técnicas construtivas iniciais. Esse trabalho demonstra a importância da preservação e reabilitação predial em patrimônios históricos como forma de resguardar a arquitetura, história e cultura de uma comunidade. Traz consigo também a caracterização pela termografia, um método não invasivo que possibilita uma melhor avaliação das anomalias apresentadas no edificado estudado.
\end{abstract}

Palavras-chave: vistoria, patologias, patrimônios, termografia, ensaio não destrutivo

Abstract: Safeguarding the historical and cultural heritage is a common interest of society, since it expresses the local cultural and economic identity. In the region of the Municipality of Curvelo/MG, the buildings are derived from the railroad base of the last century, which brought significant socioeconomic 
evolution to the region. Keeping these historic structures in a good state of conservation poses challenges inherent to conservation policies, in addition to specific knowledge of materials and retrospective construction techniques. The objective of this work is to identify pathological manifestations using thermography to characterize the historical heritage of the Curvelo/MG region, particularly Building 2, known as Lúcio Cardoso, which belongs to the urban complex of Praça Central do Brasil. The analysis of buildings of historical importance must be preceded by detailed planning. The methodological proposal consists of the application of a non-destructive test (END), namely the thermography technique. This aims to assist in building inspection, highlight the anomalies existing in the analyzed building, as well as identify its constructive characteristics and possible architectural reforms or changes. With thermography it is possible to observe some anomalies such as cracks, infiltrations, leaks, displacement of coatings or paint, disintegration of walls, among others. For this case study, the survey of information and visual data collections, surveys of previous reports, bibliographic reviews and historical architectural analysis of the building were also carried out. As a result, it was verified with the inspection and visual analysis that the building is in the process of being renovated and is in good condition, despite not having been fully preserved, its initial construction techniques. This work demonstrates the importance of building preservation and rehabilitation in historical heritage as a way of protecting the architecture, history and culture of a community. It also brings the characterization by thermography, a non-invasive method that allows a better evaluation of the anomalies presented in the studied building.

Keywords: survey, pathologies, heritage, thermography, non-destructive testing.

\section{Introdução}

A durabilidade dos edifícios está relacionada aos materiais utilizados na sua construção. Assim, habitualmente encontramos manifestações patológicas causadas por agentes físicos ou químicos que podem estar associadas a intemperismo ou ataque de fungos. Existe ainda a possibilidade de ocorrência de falha de projeto ou durante o período de construção, além de ocorrências relacionadas à durabilidade dos materiais ou carência de manutenção levando a pouca ou nenhuma conservação da estrutura. Essas anomalias, irregularidades que ocasiona a perda de desempenho da edificação, podem ser classificadas como endógenas, funcionais e exógenas (ABNT, 2020).

Em alguns casos, a sociedade utiliza o patrimônio edificado sem proteção ou manutenção mínima. Frequentemente, sofremos com a instabilidade jurídica ou a falta de recursos para proteger a integridade arquitetônica, cultural e histórica do patrimônio edificado levando a uma má preservação. Com a crescente transformação mundial, os debates em relação a proteção de patrimônios históricos ficaram cada vez mais acirrados, por se retratar de uma forma de demarcar na linha do tempo a história, cultura e sua evolução socioeconômica.

O prédio escolhido como objeto de estudo, denominado como Prédio Lúcio Cardoso (Prédio 2), faz-se fragmento do conjunto urbano da Praça Central, onde localiza-se mais 3 edificados e a antiga estação ferroviária. Os edifícios foram construídos na chegada da Rede Ferroviária Federal S/A (R.F.F.S.A) à cidade de Curvelo/MG, e teve como objetivo abrigar os trabalhadores que prestavam serviço à rede (CURVELO, 2003). Devido à grande importância da R.F.F.S.A na evolução econômica da região de Curvelo/MG, entende-se a importância de manter os prédios e as suas respectivas documentações a salvo, para manter a integridade histórica da região. No procedimento utilizado para a escolha dos edifícios para o estudo de caso, foi levada em conta a indagação histórica e cultural bem como a logística de visita para todos os integrantes da equipe.

Segundo Silva (2016), integralmente todas as edificações exigem intervenções periódicas durante o seu tempo de vida útil para que continue atendendo aos requisitos de desempenho exigidos pela NBR 15575 (ABNT, 2013). Edificações em que não ocorrem essas intervenções, ou manutenções, podem vir a sofrer com a degradação precoce de suas partes e perda de desempenho ocasionadas por má qualidade dos materiais ali usados e/ou deficiências no projeto (POSSAN; DEMOLINE, 2013).

No processo de investigação do Prédio 2, foram utilizadas as recomendações do Conselho Internacional dos Monumentos e Sítios - ICOMOS (ICOMOS, 2002) e a norma NBR 16747(ABNT, 2020) para analisar, preservar e caracterizar as manifestações patológicas encontradas. Os patrimônios, por terem um valor histórico,

Utilização da termografia na identificação de anomalias exógenas e funcionais no patrimônio histórico de Curvelo/MG. 
cultural e arquitetônico para aquele local e, considerando suas características construtivas e a idade associada, devem passar por Inspeções Prediais com maior periodicidade em relação as demais construções. A Inspeção Predial pode ser entendida como um processo sistêmico e predominante sensorial que avalia as condições técnicas, de uso, operação, manutenção e funcionalidade da edificação (ABNT, 2020). Para auxiliar nesse processo de Inspeção Predial podemos usar como auxiliares, os ensaios não destrutivos (ENDs).

Os ENDs contam com equipamentos que não põem em risco a estrutura original do edificado, sendo aconselhável a sua utilização em investigações em patrimônios e estruturas em utilização. Neste estudo de caso do Prédio 2, pertencente ao conjunto urbano da Praça central do Brasil foi utilizada a câmera termográfica como forma de complementação da inspeção predial. A técnica da termografia permite, em alguns casos, uma interpretação amplificada das manifestações patológicas visíveis e não visíveis, e têm a possibilidade de detectar com melhor precisão a intensidade das anomalias tendo potencial de auxiliar e direcionar a possíveis soluções para os danos no patrimônio.

\section{Metodologia}

Para este trabalho, a metodobologia utilizada englobou uma revisão bibliográfica acerca do tema, seleção do patrimônio estudado e a definição das técnicas para inspeção predial com auxílio da termografia. Estas etapas foram detalhadas na seção a seguir.

\subsection{Revisão bibliográfica}

Nessa etapa da metodologia foi desenvolvida uma pesquisa geral sobre os patrimônios históricos de Minas Gerais e os órgãos IPHAN (Instituto do Patrimônio Histórico e Artístico Nacional) e IEPHA (Instituto Estadual do Patrimônio Histórico e Artístico de Minas Gerais) que são responsáveis pela proteção e manutenção dos mesmos.

Em um segundo momento foi realizada uma extensa pesquisa por leis e lista de bens imóveis tombados na região de Curvelo/MG. Após o conhecimento dos imóveis tombados, foi desenvolvido um levantamento histórico com propósito de conhecer os impactos sociais e a importância da edificação na época em que foi inaugurada, para posterior escolha do imóvel objeto da análise. Dessa forma, entrou-se em contato com a Secretaria Municipal de Cultura de Curvelo/MG que disponibilizou o dossiê com o projeto arquitetônico dos patrimônios, contendo planta baixa, de locação, vistas e cortes (CURVELO, 2003). Além das documentações sobre os decretos de tombamento foram disponibilizadas também imagens da edificação antes de sofrer reforma.

\subsection{Seleção do objeto de estudo e definição das atividades}

Levando em consideração as observações de importância histórica, econômica e social, disponibilidade de documentos e a facilidade de acesso dos autores ao patrimônio, foi selecionado o Prédio Lúcio Cardoso (Prédio 2). Neste edifício realizou-se a análise que envolveu a inspeção predial e o uso da câmera termográfica da marca Flir modelo T-300, pertencente ao Programa de Pós-graduação de Engenharia Civil do Campus II - Belo Horizonte (Figura 1).

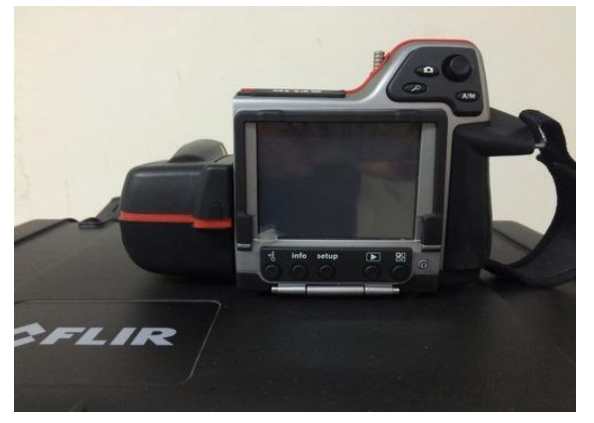

Figura 1 - Câmera termográfica utilizada.

Fonte: Autores (2020).

Utilização da termografia na identificação de anomalias exógenas e funcionais no patrimônio histórico de Curvelo/MG. 
Na Figura 2 a seguir é possível observar o conjunto de prédios da praça da estação, juntamente com o edifício escolhido como objeto de estudo (ICOMOS, 2002) e a norma ABNT NBR 16747 (ABNT, 2020).

\begin{tabular}{|l|l|}
\hline Antiga Estação Ferroviária & $\square$ \\
\hline Prédio 2 & $\square$ \\
\hline Prédio 4 & $\square$ \\
\hline
\end{tabular}

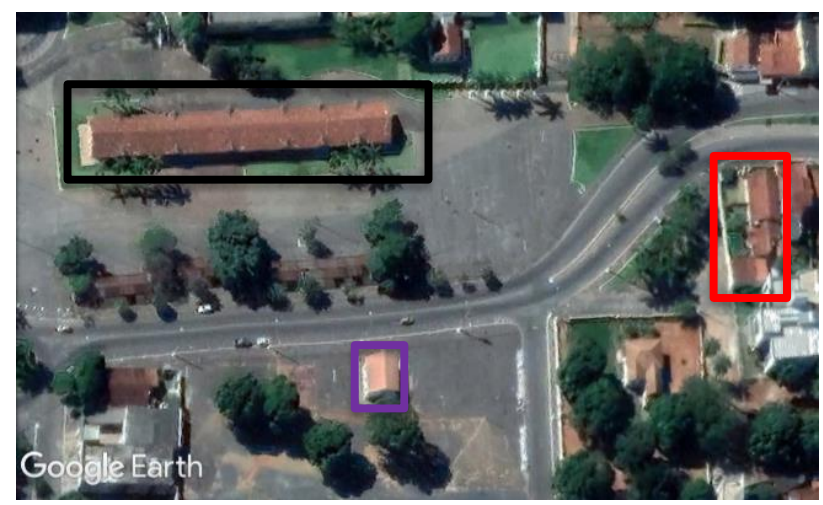

Figura 2 - Imagem aérea dos edifícios.

Fonte: Google Earth (Referência: jun 2020).

Neste trabalho serão priorizadas as análises das anomalias exógenas e funcionais. Segundo IBAPE (2012), as anomalias exógenas são irregularidade decorrentes de danos causados por terceiros e as anomalias funcionais são provenientes da degradação do imóvel, que podem estar relacionadas às falhas de manutenção e ao tempo de vida útil do edificado.

\section{Resultados}

Estão descritos na seção a seguir, os resultados obtidos neste trabalho através da revisão bibliográfica definida e a realização das inspeções prediais. Os resultados obtidos foram apresentados a seguir.

\subsection{Revisão bibliográfica}

Com a aplicação da metodologia de trabalho, obtivemos informações como: práticas adotadas pela Prefeitura Municipal na preservação (inventário e legislações), localização geográfica, última vistoria realizada, acervo fotográfico, função para qual ela foi construída, entre outras informações (geometria, forma e função, estilo arquitetônico).

Dentre os meios adotados para proteção do patrimônio histórico de Curvelo/MG, o principal deles é a Lei Municipal no 2895, de 15 de dezembro de 2014, que estabelece as normas de proteção dos bens históricos da cidade e região. A referida lei sucedeu a Lei Municipal no 1978, de 31 de dezembro de 1997, que estabelecia os parâmetros no passado.

O Prédio 2 teve suas fachadas tombadas a partir de decreto municipal no 1070, de 11 de novembro de 2002. Este patrimônio, localizado na Avenida Integração, 141, foi construído com intuito de abrigar funcionários da RFFSA (Rede Ferroviária Federal Sociedade Anônima) e na última vistoria apresentada no dossiê, que aconteceu em abril de 2002, apresentava um estado de conservação diferente do atual (Figura 3). Embora a edificação apresenta-se um bom estado de conservação, ela sofria com alguns problemas de ordem física como: trincas, partes da alvenaria que estavam faltando, vidros quebrados, apodrecimento de algumas partes das esquadrias e do telhado e desgaste da pintura, além da ambientação aos arredores da edificação estar suja (CURVELO, 2002).

A edificação trata-se de uma construção térrea, de apenas um pavimento, com planta em partido retangular. $O$ telhado é dividido em duas águas com cumeeira, telhas francesas, estrutura de madeira e beiral simples. Sua estrutura, assim como as paredes, são de alvenaria de tijolos maciços de barro. Suas esquadrias são de madeira e vidro, sendo emolduradas por ressaltos em argamassa e apresenta frontão trabalhado com detalhes em relevo, frisos e ornamentos.

Após a vistoria, a equipe técnica da Prefeitura Municipalde Curvelo/MG indicou os principais problemas encontrados, possíveis causas e diretrizes de intervenções. Como consequência dessa ação,é possivel 
afirma que a edificação se encontrava em uma má proteção. Também deve ser considerado a lei Municipal no 1978 de 31\12\1997 e pelo Decreto no1070 de 29\11\2002 e os decretos municipais vigentes, que mostram o interesse do município em conservar seu patrimônio.

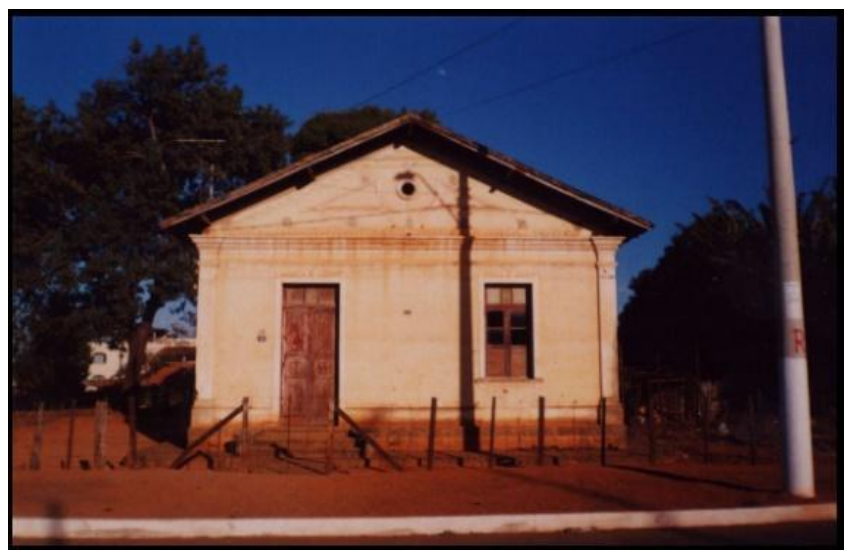

Figura 3 - Foto da fachada frontal.

Fonte: Dossiê Secretaria Municipal de Curvelo/MG (2002).

\subsection{Inspeções prediais}

Na primeira inspeção, o edifício passou por processo de inspeção visual, baseado nos critérios da NBR 16747 (ABNT, 2020). A segunda inspeção foi realizada com o auxílio da técnica da termografia e os resultados obtidos foram apresentados a seguir.

\subsubsection{Primeira inspeção}

Nessa etapa não foi utilizado o equipamento de termografia. Foram identificadas algumas manifestações patológicas que estavam pendentes, como apontado e registrado mediante relatório de inspeção feita pelos autores. A partir da inspeção de caracterização do patrimônio feita pelos autores em agosto de 2020, observou-se que a parte interna estava em processo de reforma. A edificação ainda tinha alguns componentes danificados nas esquadrias (sala e cozinha), a calçada encontrava-se irregular com alguns defeitos e na parte interna faltava pintura pós reforma. Na Figura 4 é possível observar um exemplo desses problemas, no entanto, a pintura externa estava em bom estado, o sistema elétrico, o sistema hidráulico, além do forró que encontrava-se em excelente estado.

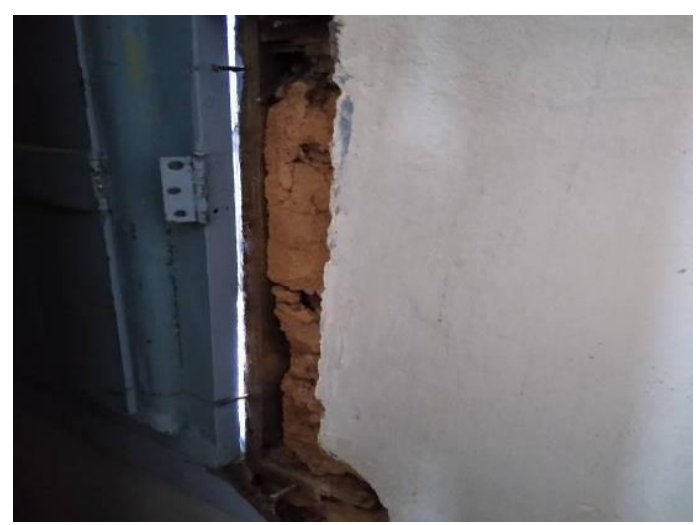

Figura 4 - Esquadria de uma das janelas.

Fonte: Autores (2020).

É possível observar e comparar somente o estado externo da edificação, já que a Prefeitura não possui em seus arquivos fotos ou informações da parte internos da edificação. Atualmente o prédio é de propriedade do Município. Percebe-se que mesmo com as intervenções periódicas realizadas pela prefeitura ainda é 
possível encontrar manifestações patológicas. Na comparação da parte externa da edificação é possível ver a evolução positiva da preservação da estrutura: a pintura encontra-se em bom estado em relação aos dados fotográficos apresentados no dossiê e, o arredor do prédio está limpo e bem conservado, o que não acontecia anteriormente. $O$ patrimônio está com suas fachadas conservadas e com nova pintura (Figura 5).

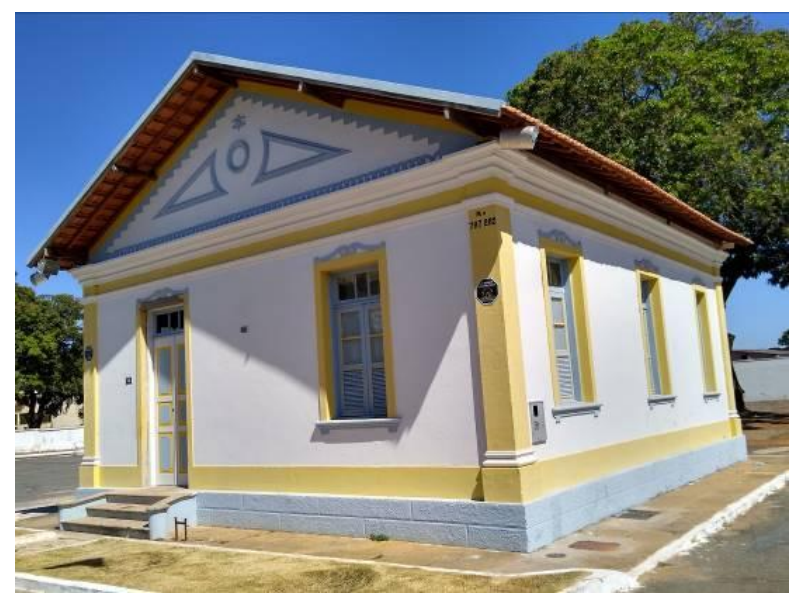

Figura 5 - Fachadas frontal e lateral da edificação.

Fonte: Autores (2020).

\subsubsection{Segunda inspeção}

A partir da segunda inspeção de caracterização do patrimônio, ocorrida em novembro de 2020, observouse que, com auxílio da termografia, pode-se identificar padrões de temperatura apresentados quando há focos de umidade em diferentes horários do dia, porém a qualidade das imagens observadas depende da incidência de insolação.

Os ensaios foram realizados pelos autores em diferentes horários do dia com intuito de registrar imagens com diferentes intensidades de calor. Com isso, foi possível observar uma provável remoção/modificação de uma janela/esquadria na fachada posterior da edificação, Figura 6.

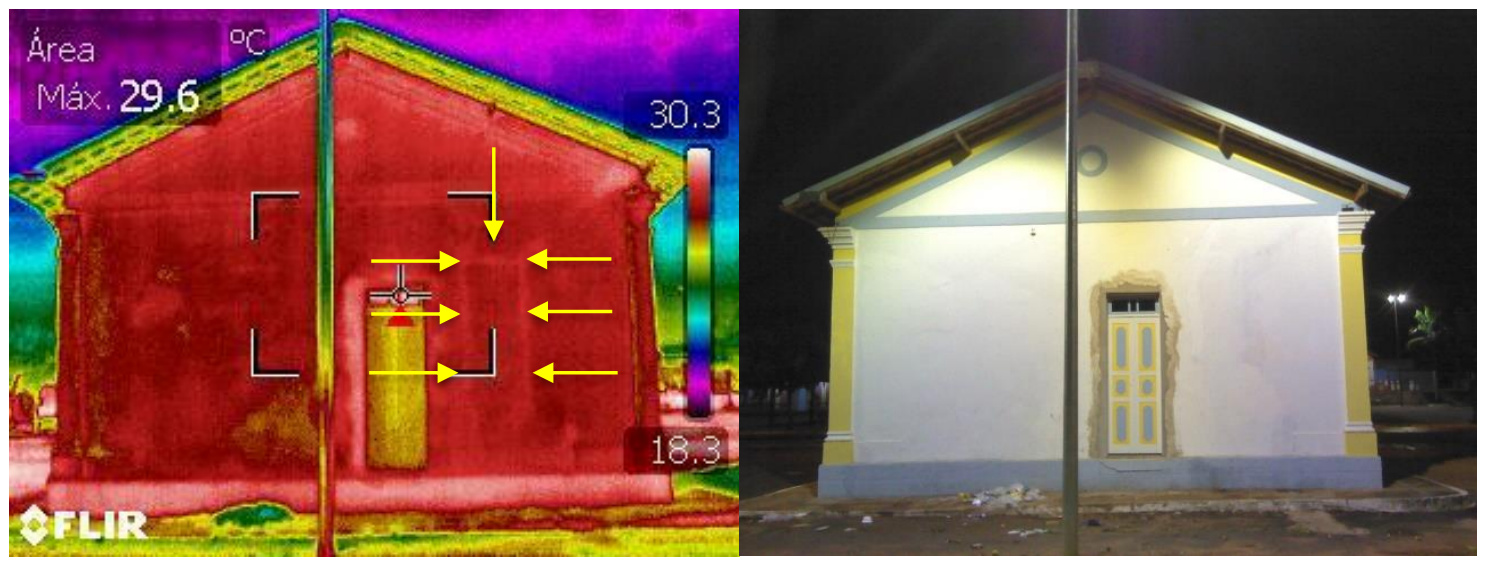

Figura 6 - Fachada posterior. Foto termográfica (esq.) e Foto real (dir.)

Fonte: Autores (2020).

Observou-se também que na Figura 7 é possível estimar que a alvenaria ultilizada nessa contrção e de tijolos maciços de barro. Mesmo já tendo essa informação através do dossiê obtido nos processos preliminares do projeto, é preciso ressaltar o alcance da termografia na caracterização do patrimônio e no auxílio à possíveis reformas e restaurações. 


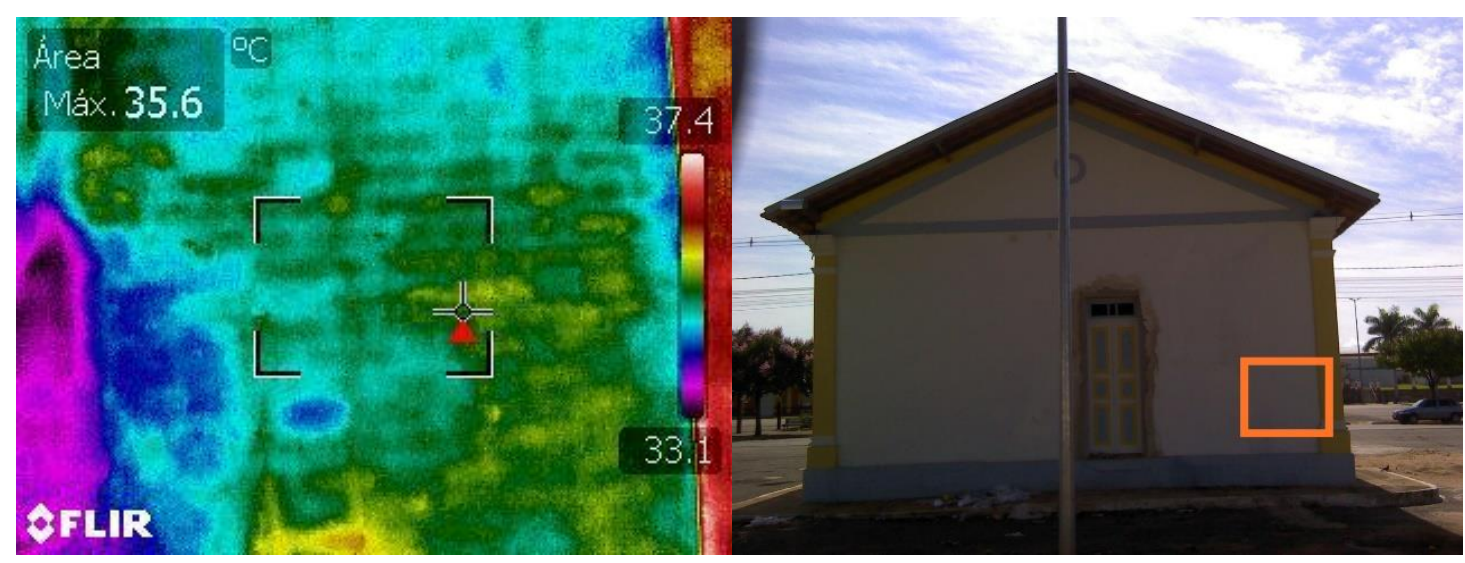

Figura 7 - Parede da fachada posterior. Foto termográfica (esq.) e Foto real de localização (dir.)

Fonte: Autores (2020).

\section{Conclusões}

Foram utilizadas neste trabalho as recomendações do Conselho Internacional dos Monumentos e Sítios ICOMOS (ICOMOS, 2002) e a norma NBR 16747 (ABNT, 2020). Em virtude dos resultados apresentados para o estudo, bem como das análises visuais realizadas sobre as imagens termográficas obtidas, é possível concluir que o Prédio Lúcio Cardoso (Prédio 2) está sendo conservado de maneira adequada, mesmo tendo alguns problemas em aberto, as esquadrias e os forros, na parte interna da edificação e entorno da edificação as trincas do passeio. Na segunda visita, verificou-se que os padrões de imagens se repetem ao longo de todo o dia durante o período de aquecimento, mas a qualidade das imagens observadas depende da incidência de insolação.

A utilização da termografia como ferramenta para auxiliar no diagnóstico de manifestações patológicas em edifícios históricos permite que haja um estudo mais detalhado e elaborado, sem que seja preciso degradar o objeto de estudo. No prédio 2, apesar de apresentar alguns problemas de ordem física, não foi identificado problemas alarmantes devido a política de conservação adotada pelo município. Mas ainda foi possível analisar algumas mudanças e particularidades do prédio através da termografia, como o fechamento de uma porta na parte de trás do patrimônio.

Pelos resultados deste trabalho, pode-se afirmar que a caracterização pela termografia se apresenta como uma ferramenta de elevado potencial a serviço das atividades de inspeção, avaliação e diagnóstico de problemas em patrimônios históricos. Sendo este um método não invasivo que possibilita uma melhor avaliação das anomalias apresentadas pelo edificado estudado.

Conclui-se com a vistoria e a análise visual que a edificação está em processo de reforma e encontra-se em bom estado de conservação, apesar de não ter sido preservada na integridade suas técnicas construtivas iniciais. Esse trabalho demostra a importância da preservação e reabilitação predial em patrimônio historicos como forma de resguarda a arquitetura, historia e cultura de uma comunidade

\section{Agradecimentos}

Agradecemos ao Centro Federal de Educação Tecnológica de Minas Gerais - CEFET-MG pelo apoio direto e indireto a este trabalho e também a Prefeitura Municipal de Curvelo/MG por permitirem a realização deste estudo e por se colocarem à disposição para auxiliar em qualquer questão necessária. Também agradecemos ao apoio do LAR - Laboratório de Avaliação e Reabilitação de Ambiente Construído do CEFETMG - Campus Curvelo, bem como a equipe do Projeto de Pesquisa PIBIC-Jr "Caracterização e avaliação do patrimônio histórico da região de Curvelo/MG" pelo incentivo a este trabalho. Pelo empréstimo do equipamento utilizado, agradecemos ao Programa de Pós-graduação de Engenharia Civil (PPGEC) do Campus II - Belo Horizonte. 


\section{Referências Bibliográficas}

ABNT - Associação Brasileira de Normas Técnicas. NBR 16747: Inspeção Predial: diretrizes, conceitos, terminologias e procedimentos. Rio de Janeiro, 2020.

ABNT - Associação Brasileira de Normas Técnicas. NBR 15424: Ensaios não Destrutivos - Termografia Terminologia. Rio de Janeiro, 2006.

Instituto Brasileiro de Avaliações e Perícias de Engenharia. Norma de Inspeção Predial Nacional. São Paulo, 2012.

ICOMOS - Comitê Científico Internacional para Análise e Restauração de Imóveis do Patrimônio Arquitetônico. Recomendações para análise, conservação e restauração estrutural do patrimônio arquitetônico. 2002. 42 p. Disponível em: < https://core.ac.uk/download/pdf/55604452.pdf >. Acesso em: $10 / 11 / 2020$

Martins, D. S., Kist L., Dos Santos, M. D., Dos Santos, M. M. A termografia infravermelha como ferramenta de auxílio nas restaurações patrimoniais com manifestações ocultas. Disponível:< https://online.unisc.br/seer/index.php/cepa/article/download/12783/7615 >. Acesso em: 30/10/2020.

Mario, M. Uso da Termografia como Ferramenta não Destrutiva para Avaliação de Manifestações Patológicas Ocultas. 2011. 60 f. Trabalho de Diplomação (Graduação em Engenharia Civil) - Departamento de Engenharia Civil, Universidade Federal do Rio Grande do Sul, Porto Alegre, 2011.

Possan, E.; Demoliner, C. A. Desempenho, durabilidade e vida útil das edificações: abordagem geral. Paraná: Revista Técnico-Científica do Crea-PR, 2013.

Prefeitura Municipal de Curvelo/MG. Lei Municipal no 1978, de 31 de dezembro de 1997, que estabelece as normas de proteção dos bens históricos da cidade e região. Disponível em: < amfcorrea.ddns.net/pmAgilNET/PGN/pgnLaiLegD.aspx?id=6423>. Acesso em: 20/10/2020.

Prefeitura Municipal de Curvelo/MG. Dossiês de Tombamento. 2003. Edificações do Conjunto de Bens Imóveis que pertenceu à Estrada de Ferro Central do Brasil. Disponível na: Sec. Municipal de Curvelo/MG.

Lista de Bens culturais protegidos: Tombados e inventariados de Curvelo/MG. Disponível em: http://curvelo.mg.gov.br/site/wp-content/uploads/2016/11/Rela\%C3\%A7\%C3\%A3o-de-bens-tombados-e-inventariadosMunic\%C3\%ADpio-de-Curvelo-1.pdf. Acesso em: 20/10/2020. 